\title{
LMPred: Predicting Antimicrobial Peptides Using Pre-Trained Language Models and Deep Learning
}

\author{
William Dee ${ }^{1}$ \\ ${ }^{1}$ Riverside Mansions, London, E1W 3TA, United Kingdom
}

\begin{abstract}
Antimicrobial peptides (AMPs) are increasingly being used in the development of new therapeutic drugs, in areas such as cancer therapy and hypertension. Additionally, they are seen as an alternative to antibiotics due to the increasing occurrence of bacterial resistance. Wet-laboratory experimental identification, however, is both time consuming and costly, so in-silico models are now commonly used in order to screen new AMP candidates. This paper proposes a novel approach of creating model inputs; using pre-trained language models to produce contextualized embeddings representing the amino acids within each peptide sequence, before a convolutional neural network is then trained as the classifier. The optimal model was validated on two datasets, being one previously used in AMP prediction research, and an independent dataset, created by this paper. Predictive accuracies of $93.33 \%$ and $88.26 \%$ were achieved respectively, outperforming all previous state-of-the-art classification models.
\end{abstract}

Sequence Analysis | Natural Language Processing | Deep Learning Correspondence: williamtimothydee@gmail.com

\section{Introduction}

Antimicrobial peptides (AMPs) are a set of naturallyoccurring molecules that exhibit a wide range of functions, including antibacterial, anticancer, antifungal and antihypertensive properties (1). When used to create therapeutic drugs, peptides are increasingly showing efficacy in terms of treatment in a variety of important areas; ranging from cancer targeting, to eliminating bacterial, viral and fungal pathogens (2).

Current cancer therapies, such as radiotherapy and chemotherapy, often elicit harmful side-effects, and cancer cell resistance to chemotherapeutic agents is a large and growing issue (3). Peptides have long been used as biomarkers in the detection and diagnosis of specific cancers, such as pancreatic, colorectal and lung (4). However, recently their use has been extended, as they have been shown to bind to specific cancerous sites and so have been used as carriers for targeted drugs (5). Certain peptides have also been shown to exhibit an inhibitory effect in cancer cells themselves (6). AMPs with anticancer properties (ACPs) are therefore promising drug development candidates due to their "high specificity, low production cost, high tumor penetration, and ease of synthesis and modification" (7), as well as their low toxicity.

Additionally, pathogenic bacteria are more frequently devel- oping multi-drug resistance $(1,8)$, rendering current antibiotic treatments ineffective. Given that AMPs are endogenous, they have shown a lower likelihood for bacteria to develop resistance to them (9), and so offer a complementary alternative to traditional drugs.

Identification of natural AMPs is therefore becoming increasingly important. However, experimental identification is both time-consuming and costly, hence the need for in-silico prediction models $(1,10)$. Previous model-based methods have utilized a range of Machine Learning approaches, including; Hidden Markov Models (11), Fuzzy K-Nearest Neighbour (FKNN) (4), Random Forest (RF) (1), Discriminant Analysis (DA) (12) and Support Vector Machines (SVM) (7, 13, 14).

Whilst these methods achieved high levels of accuracy, the feature creation steps were often extensive. Bhadra et al. (1) performed a survey of existing approaches to summarize the common data preprocessing steps. Previous research was most likely to use "compositional, physicochemical, structural properties, sequence order and the pattern of terminal residues" in order to create the final feature set.

Manavalan et al. (13) generated 20 features representing amino acid compositon, 400 relating to dipeptide composition, 5 associated with atomic composition - i.e., the frequency of each atom $(\mathrm{C}, \mathrm{H}, \mathrm{N}, \mathrm{O}$, and $\mathrm{S})$ within each peptide sequence - and 11 referring to physicochemical properties - i.e., the percentage composition of polar, hydrophobic, charged, aliphatic, aromatic, positively/negatively charged, tiny/small and large residues, as well as overall peptide mass. In total this resulted in 436 additional features.

To address the large, and often sparse, feature spaces produced by prior methods, Bhadra et al. used an approach based on the "Global Protein Sequence Descriptors" (15). This created a reduced set of 105 features which was subsequently cut to 23 using a correlation analysis based on feature importance measures. At the time this was considered the "smallest feature set for AMP prediction with high accuracy" (1). Compositional metrics, like those described above, fail to take sequence order into account - even though it is integral to the underlying function of peptides. To account for this, some prior approaches used Chou's pseudo-amino acid composition (PseAAC) (16). This generates correlation factors between each amino acid, to partly incorporate some information relating to sequence order $(4,17)$. Similarly, Evolutionary Feature Construction (EFC) (18) has also been used to detect functional signals representing nonlocal, position- 
specific interactions at the nucleotide level (19).

Two recent papers have achieved improved model accuracies by applying Natural Language Processing (NLP) techniques to create vectorized embeddings of the amino acid sequences as the sole input features $(20,21)$. The embeddings are fed into a convolutional neural network in order to classify sequences as functional AMPs or not. This has the benefit of reducing the time taken, as well as the expert biological knowledge needed, when creating model inputs. Furthermore, NLP approaches have the potential to represent richer positional information reflecting the specific locations of amino acids within a given sequence.

Veltri et al. (20) utilized the Bag of Words (BoW) method to initially assign a unique numerical token to each amino acid in a sequence. This approach can recognize basic patterns, such as which amino acids are the same, and the frequency of specific amino acids in a sequence or throughout the dataset. However, BoW fails to capture component similarity. To partially alleviate this issue, an initial embedding layer was used in the neural network architecture, to convert the discrete vectors into a continuous and dense latent space represented by a three number vector, which can then reflect more complex relationships between inputs. Wu et al. (21) used the Word2Vec Skip-Gram algorithmn which learns by being given a central word (or amino acid in this case) and then predicting the most likely words in a fixed window surrounding it, allowing it to reflect word similarity (22). However, this method still fails to convey the contextual information encoded by the position of each amino acid.

This paper proposes a novel method of creating embedding vectors - by utilizing language representation models that have been pre-trained on large protein databases to produce contextualised embeddings. Language Models (LMs) are built using the Transformer architecture (23), and have primarily been used for language-based tasks, given that they have been pre-trained on large corpora such as the 2,500 million words found in Wikipedia (24). They have been proven to outperform humans in some assessments, such as the Stanford Question Answering Dataset - a reading comprehension test (25), as well as the two NLP approaches previously mentioned. When these models are pre-trained on protein sequences, treating each amino acid as a word, and the sequence as a sentence, they have shown an ability to generalize towards understanding the "language of life" itself (26).

The LMs selected for this report are the auto-encoder models BERT (24) and T5 (27), and the auto-regressive model XLNet (28). These have already been pre-trained using the Summit supercomputer on either the UniRef100 or the UniRef50 datasets, consisting of 45 and 216 million protein sequences respectively (29). The UniRef clusters are proteins sourced from the UniProt database, with the number referring to the similarity threshold set in the CD-HIT program (30). Therefore, UniRef100 contains all UniProt sequences, whereas UniRef50 only contains sequences that do not share $>=50 \%$ identity. Additionally, BERT and T5 were also pre-trained on the Big Fat Database dataset, comprising 2,122 million sequences (31). All models were accessed via the ProtTrans
Github page (https://github.com/agemagician/ProtTrans). Bidirectional Encoder Representations from Transformers (BERT) aims to predict data from artificially corrupted inputs. It does this by adding [MASK] tokens during pretraining to replace a percentage of input words at random and it then aims to predict this masked word based on the surrounding context. In this manner it is able to capture bidirectional context. However, all masked words are predicted in parallel and independently of one another, which means that some of the overall context of a sentence can be lost during training. Also, whilst the artificial [MASK] tokens are used during pre-training, they are absent when the model is subsequently fine-tuned on a specific task, which can result in a "pretrain-finetune discrepancy" (28).

XLNet is an auto-regressive model, which functions more similarly to a feed forward neural network; these models aim to predict the next word from a set of words, given the context. However, unlike auto-encoder models, the prediction is constrained to be uni-directional. XLNet partially overcomes this drawback through permutation language modelling, whereby the model maximizes the expected log likelihood of the sequence when all possible permutations of the sequence order are considered (28). By learning context from randomly ordered sentences the resulting model is in essence bidirectional without requiring masking. Furthermore, XLNet utilizes a memory mechanism introduced by a previous auto-regressive model - Transformer-XL (32) - which allows for processing of longer contextual segments of data than BERT.

The Text-To-Text Transfer Transformer (T5) was introduced after Raffel et al. (27) studied the current landscape of transfer learning techniques for NLP and found that, generally, encoder-decoder models outperformed those only utilizing one half of the Transformer architecture. The T5 model therefore uses both parts of the Transformer, in contrast to BERT which only uses the encoder, and XLNet which only uses the decoder. T5 was also trained on a new "Colossal Clean Crawled Corpus", a comparatively clean and natural text dataset that was several magnitudes larger than many previous training datasets. There is evidence that this additional complexity, and the fact that positional encodings for each attention head can be shared across all layers, gives T5 a performance advantage over the other two LMs (33).

A convolutional neural network has been chosen as the classifier based on its success in prior peptide prediction research $(20,21)$, as well as its successful application to other areas within bioinformatics, such as mapping protein sequences to folds (34) or the prediction of RNA secondary structure (35). The convolutional layers apply filters which can interpret the spatial and temporal dependencies between amino acids that have been represented by the contextualized LM embeddings.

\section{Methods}

Datasets. Validation of the approach was performed on two datasets. One which was sourced externally and has been used in prior AMP prediction research - referred to as the 
"Veltri Dataset". The second has been constructed independently using publicly available resources - the "LMPred Dataset".

Veltri Dataset.The Veltri dataset was extracted from the Antimicrobial Peptide Scanner web page (https://www.dveltri.com/ascan/). This contains 1,778 AMP and 1,778 non-AMP samples and is split into the exact training, validation and test sets that Veltri et al. (20) used to build and evaluate their model.

LMPred Dataset. The LMPred dataset has been built from a combination of external sources. The aim was to produce the largest and most up-to-date AMP dataset possible.

The positive samples (the AMPs) have been gathered from the freely available datasets shared by Veltri et al. (20) and Bhadra et al. (1), and combined with the peer-reviewed, natural AMPs taken from the DRAMP 2.0 database (36). When duplicates were removed, these sources contributed 7,053 AMPs. The samples are then filtered by removing AMPs less than 10 amino acids in length, as well as those sharing 90\% sequence identity according to the CD-HIT online web server (30). CD-HIT is a program used to cluster proteins according to a defined similarity criterion. Applying the program to the dataset can help to eliminate bias towards particular arrangements of sequences, producing more diverse data which can be more beneficial for model training. After filtering, the remaining 3,758 AMPs were included in the LMPred dataset, being $24 \%$ more than the next largest collection used by Bhadra et al. (1).

There is little incentive to experimentally prove a peptide is non-AMP, and thus there are no large repositories of peptides that have been shown to lack desirable activities. Therefore, without access to a formal non-AMP database, negative samples were collected similarly to prior research $(4,20)$. Sequences that had been reviewed and verified were downloaded from the UniProt database (https://www.uniprot.org/). The data was then filtered according to the following criteria:

- Any duplicate entries were removed.

- Only samples whose subcellular location was cited as "cytoplasm" were retained to ensure the origination was similar to the AMP samples.

- Any samples labelled as specifically having the activities: "antimicrobial", "antibiotic", "antiviral", "antifungal", "effector" or "excreted", were omitted.

- Datapoints with fewer than 10 amino acids, and more than 255 amino acids were removed. The remaining samples then mirrored the range of AMP sequence lengths in the positive sample dataset.

- Sequences which contained unnatural amino acids (Z, $\mathrm{B}, \mathrm{J}, \mathrm{O}, \mathrm{U}$ or $\mathrm{X}$ ) were removed.

- Finally, sequences were screened for similarity using the CD-HIT program, using a $40 \%$ similarity threshold. The threshold can be stricter for non-AMPs given the larger number of available sequences, and this ensures a more diverse dataset overall.

Previous research found that if the negative sample sequence length distribution matched that of the positive samples, this resulted in the highest classification accuracy models (20). Therefore 3,758 non-AMPs were selected from the remaining pool of 33,722 to compile a final dataset which matched this criterion. The dataset was then split using sklearn's "train_test_split" function (https://scikit-learn.org/); $40 \%$ of the data was set aside for training, $20 \%$ for validation and the remaining $40 \%$ for testing. The splitting was performed on a stratified basis and the distribution of samples across these partitions can be found in the supplementary information. The dataset has been made freely available to download at: https://github.com/williamdee1/LMPred_AMP_Dataset.

Language Model Embeddings. Instructions were followed on the ProtTrans Github page for how to create word embeddings using each LM. An overview of this process is as follows:

- Download the specific tokenizer and pre-trained LM hosted on the ProtTrans Rostlab server.

- Convert the sequences of amino acids into a list and add spaces in-between each amino acid.

- Any unnatural amino acids ("U, Z, O, B, J") are mapped to "X".

- The sequence IDs are encoded in batches by the tokenizer, padding with zeros to a specified max length so all inputs are the same length.

- Torch tensors representing the input IDs and the mask used for the attention mechanism are created.

- The embeddings are generated in batches of ten to ensure memory constraints are not breached and the output is saved as a numpy array.

Certain LMs produce special tokens, such as [CLS] or [SEP] tokens, that are included in the embedding array. The CLS token is created by some models to be used as an intelligent average 1-dimensional vector summarizing the full 2dimensional embedding. It is often used as the input for NLP classification tasks. The SEP token separates any special tokens from the embeddings. In this project the full embeddings were used as not all LMs produce CLS tokens and this ensures greater comparability across results. Additionally, using the full embeddings ensures valuable information isn't lost and, instead, the neural network can decide how to screen the data through use of the filters, kernel and max pooling layers.

An example of the embedding output is shown in Figure 1.

Model Architecture. A convolutional neural network $(\mathrm{CNN})$ was chosen as the classifier for this paper, and was built using the Keras framework (https://keras.io/), which utilizes the Tensorflow (37) back-end. 


\section{Embedding Output Example}

Embedding Output:

$2 \mathrm{D}$ vector with shape (max input length, 1,024), where max input length is equal to the longest sequence found in the dataset.

Example sequence: "MQLSAPHCKKL"

$$
\begin{aligned}
& \text { "M" }\left[\begin{array}{lll}
{[0.1120588,} & 0.1051234,-0.00387062, \ldots, & 0.01635456,
\end{array}\right. \\
& \text { [0.02171638, } 0.02049154] \text {, }
\end{aligned}
$$

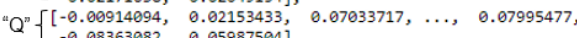

$$
\begin{aligned}
& \text { "L" }[[0.00674855,-0.01097985 \text {, }
\end{aligned}
$$

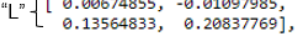

$$
\begin{aligned}
& {\left[\begin{array}{ll}
10 . \\
0 . & ,
\end{array}\right.} \\
& {\left[\begin{array}{lll}
0 . & 0 . \\
0 . & 0 .
\end{array}\right.} \\
& \text { [ } \bullet \text {. } \\
& \begin{array}{ll} 
& 0 . \\
0 . & 0 . \\
\theta & 0 . \\
\theta & 0 .
\end{array} \\
& \begin{array}{l}
\text { j, } \\
\text { j, } \\
\text { j] }
\end{array} \\
& , \ldots, 0 \\
& , \ldots, \theta \text {. } \\
& , \ldots, 0 \text {. }
\end{aligned}
$$

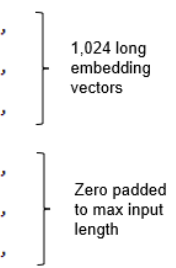

Fig. 1. An example of how the sequence 'MQLSAPHCKKL' would be represented after applying the pre-trained language models to create an embedding vector.

Two different architectures were tested for each LM. Since the models are used to produce word embeddings there is no need for an initial embedding layer in the CNN, however the number of convolutional, max pooling, dense and batch normalization layers were altered to investigate the impact on performance. A more basic configuration with one convolutional, one max pooling, one batch normalization and then a dense sigmoidal output layer was found to produce the highest classification accuracy model for the BERT UniRef100, BERT BFD and T5 BFD embeddings. A more complex architecture employing two convolutional, two max pooling, two batch normalization, two dense and two dropout layers before the final dense sigmoid output layer, was found to work best for the T5 UniRef50 and XLNet LM embeddings. An illustration of these architectures can be found in Figure 2.

Hyperparameter Tuning. For each set of LM embeddings, tuning was performed independently to ascertain the optimal hyperparameters for each resulting CNN model. The tuning was performed using the Keras Tuner (https://keras.io/keras_tuner) which provides a framework to apply different search algorithms. Both the Hyperband (38) and the Bayesian Optimization (39) tuning algorithms were used. Utilizing the two methods in combination has been shown to outperform using them separately as, "bandit-based approaches" (like Hyperband) "lack guidance", whereas Bayesian optimization across the entire search space can be "computationally infeasible" (40). The tuning uses only the training and validation partitions of the data.

Tuning was performed for a maximum of 10 epochs for Hyperband, and 15 for Bayesian Optimization, with an early stopping callback after 6 epochs without a decrease in validation loss, and a reduction of the learning rate by a factor of $1 \mathrm{e}^{-1}$ after 4 epochs without a decrease. The optimal parameters were the ones which produced the model with the lowest validation loss during training. The entire search space is detailed in Table 1.

Model training and evaluation. Once hyperparameters were selected for each LM-embedding approach, each CNN model was trained for 30 epochs with the optimizer loss function set to "binary_crossentropy" and metrics set to "accu-

\section{Convolutional Neural Network Architectures}

One Convolutional Layer Model:

\begin{tabular}{|c|c|c|}
\hline Layer & Hyperparameter & Values \\
\hline Convolutional & Filter & $\operatorname{Min}=32, \operatorname{Max}=512$, Step $=32$ \\
\hline Convolutional & Kernel & $\operatorname{Min}=3, \operatorname{Max}=33$, Step $=2$ \\
\hline Convolutional & Weight initializer & 'RandomNormal', 'HeNormal' \\
\hline Convolutional & Activation Function & 'ReLU' \\
\hline Max. pooling & Pool size & $\operatorname{Min}=2, \operatorname{Max}=14$, Step $=2$ \\
\hline Max. pooling & Pool strides & $\operatorname{Min}=256, \operatorname{Max}=1024$, Step $=128$ \\
\hline First Dense & Units & $\operatorname{Min}=256, \operatorname{Max}=1024$, Step $=128$ \\
\hline Second Dense & Units & $\operatorname{Min}=32, \operatorname{Max}=256$, Step $=32$ \\
\hline Dropout & Dropout Rate & $\operatorname{Min}=0.0, \operatorname{Max}=0.5$, Step $=0.1$ \\
\hline Optimizer & Learning Rate & Min $=1 \mathrm{e}^{-7}, \operatorname{Max}=1 \mathrm{e}^{-3}$, Step $=1 \mathrm{e}^{-1}$ \\
\hline Optimizer & Optimizer & 'Adam', 'SGD' \\
\hline
\end{tabular}

Found to be optimal for BERT (UniRef 100), BERT (BFD) and T5 (BFD).

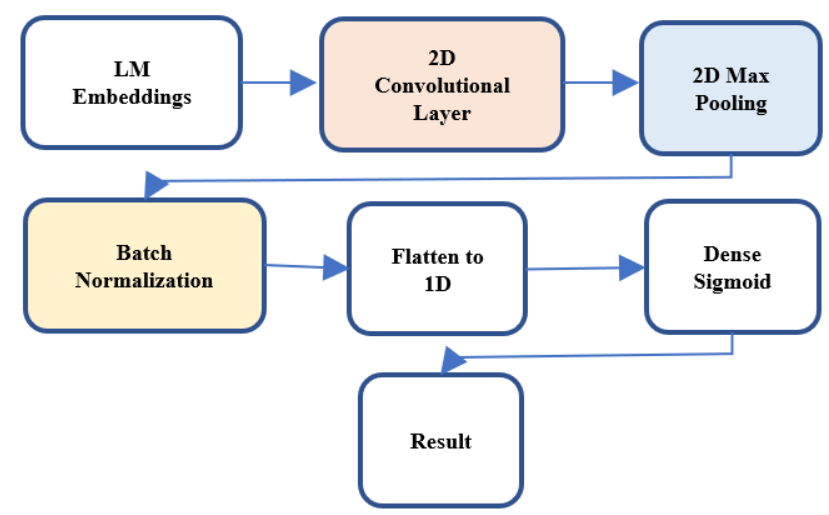

Two Convolutional Layer Model:

Found to be optimal for T5 (UniRef 50) and XLNet (UniRef100).

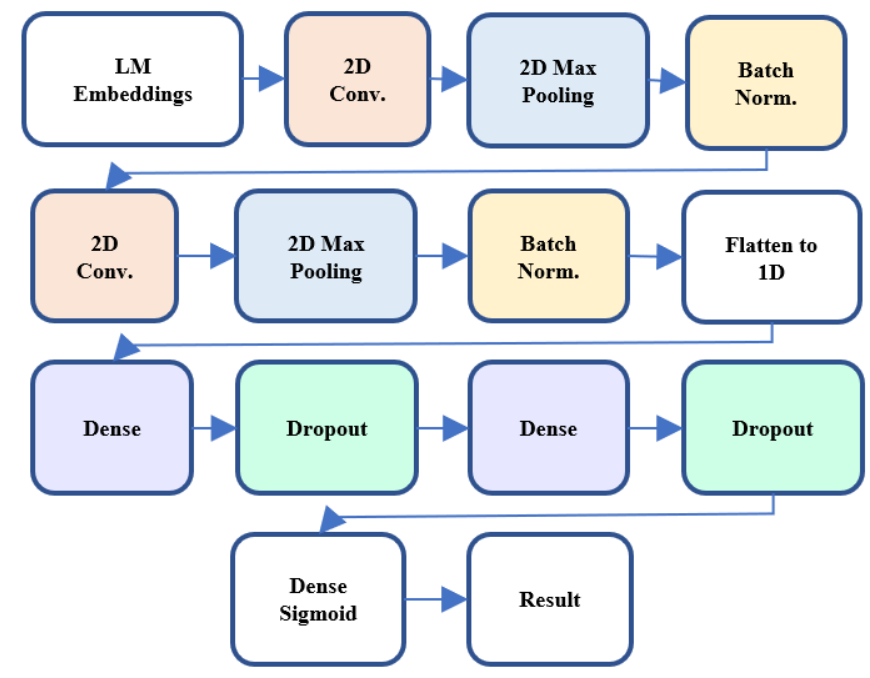

Fig. 2. An illustration of the two CNN architectures used with the different language model embedding inputs.

Table 1. Hyperparameter Search Space

racy". Early stopping was set to 12 epochs, and the learning rate was reduced by a factor of $1 \mathrm{e}^{-1}$ after 4 epochs without an improvement in validation loss. The model checkpoint callback saved the model with the lowest validation loss during 
training.

In terms of evaluation, each model was tested for accuracy (Ac.) on the test set for both the Veltri and the LMPred datasets. Accuracy is considered the goal metric as identifying positive and negative samples correctly is equally important. Additionally, sensitivity (Sn.), specificity (Sp.), Matthew's correlation coefficient (MCC) and the area under the ROC curve (AUC) were also calculated to provide a full overview of model performance. These metrics were calculated as follows using the number of true positive (TN), true negative (TN), false positive (FP) and false negative (FN) predictions:

$$
\begin{gathered}
S n .=\frac{T P}{T P+F N} \\
S p .=\frac{T N}{T N+F P} \\
A c .=\frac{T P+T N}{T P+T N+F P+F N} \\
M C C=\frac{(T P \times T N)-(F P \times F N)}{\sqrt{(T P+F P)(T P+F N)(T N+F P)(T N+F N)}}
\end{gathered}
$$

The area under the ROC curve (AUC) was calculated using the sklearn metrics package.

The results were compared to that of the available webserver models, produced by previous state-of-the-art AMP prediction papers, when provided the same test sets. These include; the CAMP server's artificial neural network, support vector machine, discriminant analysis and random forest models (12), AmPEP's random forest (1), iAMP-2L's fuzzy Knearest neighbour (4), iAMPpred's support vector machine (17) and Veltri's CNN model (20).
Models using two convolutional layers (T5 UniRef50 and XLNet) took 107 seconds per epoch to train and $166 \mathrm{sec}-$ onds to load and predict the 3,007 test samples in the LMPred dataset. The size of those models was approximately $1.16 \mathrm{~GB}$. This is compared to 35 seconds per training epoch, 34 seconds for testing and 5.2MB in size, for the models using one convolutional layer (BERT UniRef100, BERT BFD and T5 UniRef100).

\section{Results}

\section{Model performance.}

Veltri Dataset. Table 2 shows the performance of the CNN models, using the different LM embeddings as inputs, when tested on the Veltri dataset. These results have been compared with the results published in (20)'s AMP peptide prediction paper, including the metrics from that paper's own method, as well as the various webserver models available at that time.

The best performing model - T5 pre-trained on the UniRef50 database - achieved an accuracy of $93.33 \%$, being $2.55 \%$ higher than that the previous state-of-the-art accuracy results of $91.01 \%$ on the same dataset. The T5 UniRef50 model also achieved state-of-the-art MCC and auROC metrics, only being bested on specificity by the T5 BFD model, and on sensitivity by the CAMP Random Forest webserver model (12). The auto-regressive model, XLNet, underperformed the two auto-encoder (BERT and T5) LM-based approaches, as well as the approach used by Veltri et al. (20). This underperformance was noted across every metric. However, all models using NLP techniques to create embeddings, and convolutional neural networks as the classifier, displayed higher accuracies than those using biological information and machine learning models.

Replicating the state-of-the-art approach. In order to en-

Table 2. Comparison with state-of-the-art methods - Veltri Dataset

sure the most robust comparison with the current state-of-theart method for AMP prediction, a replica of the CNN built by Veltri et al. (20) was constructed. Firstly, it was tested on the Veltri dataset, and its efficacy was compared to that of the original paper, to verify it had been replicated correctly. This approach was then tested on the LMPred test set, having been trained using the additional training and validation samples provided by that dataset, which the Veltri webserver model has not had access to. Lastly, since some positive samples in the LMPred dataset were sourced from the dataset used by (20), the webserver model will have been trained on 457 of the samples it is then asked to predict. Metrics will therefore also be included showing the efficacy of the Veltri webserver model at predicting only unseen data.

Technical settings. Creating word embeddings, as well as hyperparameter tuning, training and evaluation of the CNN models was performed using Google Colab Pro (https://research.google.com/colaboratory/). A Tesla P100PCIE-16GB GPU was used, as well as up to 24GB of RAM and $150 \mathrm{~GB}$ of disk resources.

\begin{tabular}{llccccc}
\hline Approach & Method & Sn. (\%) & Sp. (\%) & Ac. (\%) & MCC & AUC (\%) \\
\hline \multicolumn{7}{l}{ Models results from } \\
the Veltri & et al. $(20)$ & paper: & & & \\
AntiBP2 & SVM & 87.91 & 90.80 & 89.37 & 0.7876 & 89.36 \\
CAMP & ANN & 82.98 & 85.09 & 84.04 & 0.6809 & 84.06 \\
CAMP & DA & 87.08 & 80.76 & 83.92 & 0.6797 & 89.97 \\
CAMP & RF & $\mathbf{9 2 . 7 0}$ & 82.44 & 87.57 & 0.7554 & 93.63 \\
CAMP & SVM & 88.90 & 79.92 & 84.41 & 0.6910 & 90.63 \\
iAMP-2L & FKNN & 83.99 & 85.86 & 84.90 & 0.6983 & 84.90 \\
IAMPred & SVM & 89.33 & 87.22 & 88.27 & 0.7656 & 94.44 \\
gkmSVM & SVM & 88.34 & 90.59 & 89.46 & 0.7895 & 94.98 \\
Veltri & CNN & 89.89 & 92.13 & 91.01 & 0.8204 & 96.48 \\
\hline Models created by & this paper: & & & & & \\
Veltri (Replica) & CNN & 88.48 & 92.70 & 90.60 & 0.8125 & 96.12 \\
BERT (Uni 100) & CNN & 90.31 & 93.82 & 92.06 & 0.8418 & 97.29 \\
BERT (BFD) & CNN & 91.99 & 92.13 & 92.06 & 0.8413 & 97.55 \\
T5 (Uni 50) & CNN & 92.28 & 94.38 & $\mathbf{9 3 . 3 3}$ & $\mathbf{0 . 8 6 6 8}$ & $\mathbf{9 7 . 8 9}$ \\
T5 (BFD) & CNN & 88.62 & $\mathbf{9 5 . 7 9}$ & 92.21 & 0.8463 & 97.41 \\
XLNet (Uni 100) & CNN & 88.48 & 91.01 & 89.75 & 0.7952 & 95.78 \\
\hline
\end{tabular}

Note: The performance of the webserver models and the Veltri et al. model was sourced from the original Veltri et al. (20) paper. This has been compared with the models built in this paper - being the replica of the Veltri model as well as the models built using different language model embeddings. 
Table 3. Comparison with state-of-the-art methods - LMPred dataset

\begin{tabular}{|c|c|c|c|c|c|c|}
\hline Approach & Method & Sn. $(\%)$ & Sp. $(\%)$ & Ac. $(\%)$ & $\mathrm{MCC}$ & $\operatorname{AUC}(\%)$ \\
\hline \multicolumn{7}{|c|}{ External Webserver Models: } \\
\hline AmPEP & $\mathrm{RF}$ & 56.62 & 43.48 & 50.05 & 0.1050 & $\mathrm{n} / \mathrm{a}^{*}$ \\
\hline CAMP & ANN & 77.51 & 72.54 & 75.02 & 0.5011 & $\mathrm{n} / \mathrm{a}^{*}$ \\
\hline CAMP & DA & 81.04 & 71.41 & 76.22 & 0.5269 & 80.16 \\
\hline CAMP & $\mathrm{RF}$ & 84.70 & 72.74 & 78.72 & 0.5785 & 83.05 \\
\hline CAMP & SVM & 83.03 & 71.88 & 77.45 & 0.5525 & 80.27 \\
\hline iAMP-2L & FKNN & 76.18 & 74.60 & 75.39 & 0.5079 & $\mathrm{n} / \mathrm{a}^{*}$ \\
\hline IAMPred & SVM & 89.55 & 55.92 & 72.73 & 0.4828 & 82.28 \\
\hline Veltri & $\mathrm{CNN}$ & 90.09 & 66.95 & 78.52 & 0.5863 & 86.45 \\
\hline Veltri (Unseen**) & $\mathrm{CNN}$ & 86.42 & 66.95 & 74.94 & 0.5277 & 84.42 \\
\hline \multicolumn{7}{|c|}{ Models created by this paper: } \\
\hline Veltri (Replica) & $\mathrm{CNN}$ & 75.98 & 85.11 & 80.55 & 0.6134 & 85.82 \\
\hline BERT (Uni 100) & $\mathrm{CNN}$ & 84.43 & 84.91 & 84.67 & 0.6934 & 90.84 \\
\hline BERT (BFD) & $\mathrm{CNN}$ & 86.83 & 88.16 & 87.50 & 0.7500 & 93.58 \\
\hline T5 (Uni 50) & $\mathrm{CNN}$ & 88.89 & 87.63 & 88.26 & 0.7653 & 94.66 \\
\hline T5 (BFD) & $\mathrm{CNN}$ & 86.16 & 88.96 & 87.56 & 0.7515 & 93.68 \\
\hline XLNet (Uni 100) & $\mathrm{CNN}$ & 82.63 & 78.92 & 80.78 & 0.6160 & 88.87 \\
\hline
\end{tabular}

Note: Results produced when testing models created within this paper, as well as external webserver models, on the LMPred test dataset.

$*_{n / a}$ : The web server did not output prediction probabilities, so AUC could not be calculated.

**Unseen: Refers to only the samples not already seen by the Veltri webserver model being submitted as the part of the LMPred test set.

LMPred Dataset. Table 3 shows the performance of the CNN models when tested on the LMPred dataset, compared to the available webserver model predictions when provided the same test data. Similar results were found to Section 3.1.1, with the T5 auto-encoder language model, pre-trained on UniRef50, producing embeddings that resulted in the CNN with the highest accuracy $(88.26 \%)$.

The performance difference between the models created by this paper and the state-of-the-art webserver models was more noticeable, likely due to the increased number of samples (111\% more than the Veltri dataset) leading to a higher difficulty classification task. The T5 UniRef50 CNN produced $12.4 \%$ higher accuracy than the Veltri webserver model, which increased to a $17.8 \%$ gap when the Veltri webserver was only presented with unseen test data.

To differentiate between the impact of the increased number of training and validation samples, and the embedding approach taken, a replica of Veltri's approach was built, utilizing the training and validation data of the LMPred dataset. This approach resulted in an uplift of $2.6 \%$ versus the Veltri webserver model, but still fell $9.6 \%$ short of the best LMbased CNN's accuracy - implying that this is the true performance difference between approaches for this dataset.

Consistent with the results found for the Veltri dataset, all models leveraging NLP techniques and CNN's scored higher accuracies than those utilizing specialist biological feature sets and traditional machine learning models. The T5 model trained on the BFD also displayed the highest specificity, whilst the Veltri and the IAMPred (17) webserver models produced the highest sensitivity. The XLNet embeddings proved to be inferior to both BERT and T5 and did not perform significantly better than the replica Veltri model.

\section{Discussion}

This paper has proposed a novel method for producing model inputs for classifying AMPs. By utilizing the contextualized embeddings produced by pre-trained language models, the resulting convolutional neural network achieved improved classification accuracy compared to the existing state-of-theart methods across two datasets. This provides further evidence that NLP techniques can replicate some of the "language of life" which previously required extensive time and biological knowledge in the feature engineering stages to represent.

The results support the research of Elnaggar et al. (33) who found that true bi-directionality of contextual embedding was extremely important for protein structure prediction. This implies that the structure of amino acids is important bidirectionally and it is likely that the permutation modelling that XLNet uses, whilst it can work for sentences, is not so applicable in the case of peptide or protein sequences. The more distant dependency modelling that XLNet allows appears to be less beneficial in this use case, as the BERT-based embeddings do not suffer from lacking it.

Furthermore, this research also supported Elnaggar et al. (33)'s results that found T5 to be the superior model for prediction - as the T5 model trained on UniRef50 generated the highest accuracy metric when tested on both datasets. This demonstrates the benefits of using the whole transformer architecture to build the pre-trained language model, rather than just the encoder (BERT) or decoder (XLNet).

Also similar to Elnaggar et al. (33)'s findings, there was no conclusive evidence that pre-training the LMs on larger datasets resulted in embeddings that improved predictive accuracy. On both the Veltri and the LMPred dataset, the T5 model trained on the BFD produced lower accuracy than the one trained on the UniRef50 dataset. The BERT BFD model did outperform the BERT UniRef100 model, but only on the larger, LMPred dataset. More diverse pre-training datasets may prove to be the optimal approach for this problem. This is implied by the results, as UniRef50 only includes the sequences from UniProt that do not share more than $50 \%$ sequence similarity. Additionally, the T5 model is also trained on a large, diverse "cleaned" corpus, compared to the smaller and more uniform corpora used to initially produce BERT. The abundance of noise in these databases, i.e., in UniRef100 caused by duplicated sequences, may have proved detrimental to overall learning.

Future research could investigate the effectiveness of the pre-trained language models not considered in scope by this paper. These include the ELECTRA and ALBERT models, as well as the Transformer-XL model when it is released on the ProtTrans Github page (https://github.com/agemagician/ProtTrans). This work would likely provide further support to the evidence found in this paper that auto-encoder models produce more contextrich embeddings which can be more effectively used as inputs into CNN models predicting AMPs. It would be interesting to note whether these performance trends are similar across AMPs with differing activities (i.e. anticancer compared to 
bioRxiv preprint doi: https://doi.org/10.1101/2021.11.03.467066; this version posted November 8, 2021. The copyright holder for this preprint (which was not certified by peer review) is the author/funder, who has granted bioRxiv a license to display the preprint in perpetuity. It is made available under aCC-BY-NC-ND 4.0 International license.

antihypertensive peptides).

Further work could also split the AMPs into explicit cohorts, i.e., based on sequence length, or the frequency of specific amino acids. This research may reveal that different language models excel at predicting different cohorts, a hypothesis that may be supported by the evidence that longer-term dependency modelling is more important in the case of longer sentences for NLP tasks, or in this case - longer sequences.

\section{Acknowledgements}

I would like to thank Ahmad Abu-Khazneh for his feedback and guidance during the completion of this project.

\section{Funding}

The author received no financial support for the research, authorship, and/or publication of this paper.

\section{Conflicts of Interest: None declared.}

\section{Bibliography}

1. Pratiti Bhadra, Jielu Yan, Jinyan Li, Simon Fong, and Shirley W. I. Siu. AmPEP: Sequencebased prediction of antimicrobial peptides using distribution patterns of amino acid properties and random forest. Scientific Reports, 8(1):1697, January 2018. ISSN 2045-2322. doi: 10.1038/s41598-018-19752-w. Number: 1 Publisher: Nature Publishing Group.

2. Jyothi Thundimadathil. Cancer Treatment Using Peptides: Current Therapies and Future Prospects. Journal of Amino Acids, 2012:e967347, December 2012. ISSN 2090-0104. doi: 10.1155/2012/967347. Publisher: Hindawi.

3. Magali Rebucci and Carine Michiels. Molecular aspects of cancer cell resistance to chemotherapy. Biochemical Pharmacology, 85(9):1219-1226, May 2013. ISSN 00062952 doi: 10.1016/j.bcp.2013.02.017.

4. Xuan Xiao, Pu Wang, Wei-Zhong Lin, Jian-Hua Jia, and Kuo-Chen Chou. iAMP-2L: A twolevel multi-label classifier for identifying antimicrobial peptides and their functional types. Analytical Biochemistry, 436(2):168-177, May 2013. ISSN 0003-2697. doi: 10.1016/j.ab. 2013.01.019.

5. Anxin Wang, Lisha Chen, Kefeng Pu, and Yimin Zhu. Identification of stem-like cells in nonsmall cell lung cancer cells with specific peptides. Cancer Letters, 351(1):100-107, August 2014. ISSN 1872-7980. doi: 10.1016/j.canlet.2014.05.004.

6. Srinivas J. Rayaprolu, Navam S. Hettiarachchy, Pengyin Chen, Arvind Kannan, and Andronikos Mauromostakos. Peptides derived from high oleic acid soybean meals inhibit colon, liver and lung cancer cell growth. Food Research International, 50(1):282-288, January 2013. ISSN 0963-9969. doi: 10.1016/j.foodres.2012.10.021.

7. Atul Tyagi, Pallavi Kapoor, Rahul Kumar, Kumardeep Chaudhary, Ankur Gautam, and G. P. S. Raghava. In Silico Models for Designing and Discovering Novel Anticancer Peptides. Scientific Reports, 3(1):2984, October 2013. ISSN 2045-2322. doi: 10.1038/srep02984. Number: 1 Publisher: Nature Publishing Group.

8. World Health Organization. Antimicrobial resistance: global report on surveillance. World Health Organization, Geneva, Switzerland, 2014. ISBN 978-92-4-156474-8. OCLC: ocn880847527.

9. H. G. Boman. Antibacterial peptides: basic facts and emerging concepts. Journal of Internal Medicine, 254(3):197-215, 2003. ISSN 1365-2796. doi: https://doi.org/10.1046/ j.1365-2796.2003.01228.x. _eprint: https://onlinelibrary.wiley.com/doi/pdt/10.1046/j.13652796.2003.01228.x.

10. Feng-Min Li and Xiao-Qian Wang. Identifying anticancer peptides by using improved hybrid compositions. Scientific Reports, 6(1):33910, September 2016. ISSN 2045-2322. doi: 10.1038/srep33910. Number: 1 Publisher: Nature Publishing Group.

11. Christopher D. Fjell, Robert E.W. Hancock, and Artem Cherkasov. AMPer: a database and an automated discovery tool for antimicrobial peptides. Bioinformatics, 23(9):1148-1155, May 2007. ISSN 1367-4803. doi: 10.1093/bioinformatics/btm068.

12. Shaini Thomas, Shreyas Karnik, Ram Shankar Barai, V. K. Jayaraman, and Susan IdiculaThomas. CAMP: a useful resource for research on antimicrobial peptides. Nucleic Acids Research, 38(Database issue):D774-D780, January 2010. ISSN 0305-1048. doi: 10.1093/ nar/gkp1021.

13. Balachandran Manavalan, Shaherin Basith, Tae Hwan Shin, Sun Choi, Myeong Ok Kim, and Gwang Lee. MLACP: machine-learning-based prediction of anticancer peptides. Oncotarget, 8(44):77121-77136, August 2017. ISSN 1949-2553. doi: 10.18632/oncotarget.20365.

14. Aarti Garg and Dinesh Gupta. VirulentPred: a SVM based prediction method for virulent proteins in bacterial pathogens. BMC Bioinformatics, 9(1):62, January 2008. ISSN 14712105. doi: 10.1186/1471-2105-9-62.

15. I Dubchak, I Muchnik, S R Holbrook, and S H Kim. Prediction of protein folding class using global description of amino acid sequence. Proceedings of the National Academy of Sciences of the United States of America, 92(19):8700-8704, September 1995. ISSN 0027-8424.
16. K. C. Chou. Prediction of protein cellular attributes using pseudo-amino acid composition. Proteins, 43(3):246-255, May 2001. ISSN 0887-3585. doi: 10.1002/prot.1035.

17. Prabina Kumar Meher, Tanmaya Kumar Sahu, Varsha Saini, and Atmakuri Ramakrishna Rao. Predicting antimicrobial peptides with improved accuracy by incorporating the compositional, physico-chemical and structural features into Chou's general PseAAC. Scientific Reports, 7(1):42362, February 2017. ISSN 2045-2322. doi: 10.1038/srep42362. Number: 1 Publisher: Nature Publishing Group.

18. Uday Kamath, Kenneth De Jong, and Amarda Shehu. Effective Automated Feature Construction and Selection for Classification of Biological Sequences. PLOS ONE, 9(7):e99982, July 2014. ISSN 1932-6203. doi: 10.1371/journal.pone.0099982. Publisher: Public Library of Science.

19. Daniel Veltri, Uday Kamath, and Amarda Shehu. Improving Recognition of Antimicrobial Peptides and Target Selectivity through Machine Learning and Genetic Programming. IEEE/ACM Transactions on Computational Biology and Bioinformatics, 14(2):300313, March 2017. ISSN 1557-9964. doi: 10.1109/TCBB.2015.2462364. Conference Name: IEEE/ACM Transactions on Computational Biology and Bioinformatics.

20. Daniel Veltri, Uday Kamath, and Amarda Shehu. Deep learning improves antimicrobial peptide recognition. Bioinformatics, 34(16):2740-2747, August 2018. ISSN 1367-4803. doi: 10.1093/bioinformatics/bty179.

21. Chuanyan Wu, Rui Gao, Yusen Zhang, and Yang De Marinis. PTPD: predicting therapeutic peptides by deep learning and word2vec. BMC Bioinformatics, 20(1):456, September 2019. ISSN 1471-2105. doi: 10.1186/s12859-019-3006-z.

22. Tomas Mikolov, Kai Chen, Greg Corrado, and Jeffrey Dean. Efficient Estimation of Word Representations in Vector Space. arXiv:1301.3781 [cs], September 2013. arXiv: 1301.3781.

23. Ashish Vaswani, Noam Shazeer, Niki Parmar, Jakob Uszkoreit, Llion Jones, Aidan N. Gomez, Lukasz Kaiser, and Illia Polosukhin. Attention Is All You Need. arXiv:1706.03762 [cs], December 2017. arXiv: 1706.03762.

24. Jacob Devlin, Ming-Wei Chang, Kenton Lee, and Kristina Toutanova. BERT: Pre-training of Deep Bidirectional Transformers for Language Understanding. arXiv:1810.04805 [cs], May 2019. arXiv: 1810.04805

25. SQUAD 2.0. The Stanford Question Answering Dataset, 2021.

26. Ahmed Elnaggar, Michael Heinzinger, Christian Dallago, Ghalia Rehawi, Yu Wang, Llion Jones, Tom Gibbs, Tamas Feher, Christoph Angerer, Martin Steinegger, Debsindhu Bhowmik, and Burkhard Rost. ProtTrans: Towards Cracking the Language of Life's Code Through Self-Supervised Learning. preprint, Bioinformatics, July 2020.

27. Colin Raffel, Noam Shazeer, Adam Roberts, Katherine Lee, Sharan Narang, Michael Matena, Yanqi Zhou, Wei Li, and Peter J. Liu. Exploring the Limits of Transfer Learning with a Unified Text-to-Text Transformer. arXiv:1910.10683 [cs, stat], July 2020. arXiv: 1910.10683.

28. Zhilin Yang, Zihang Dai, Yiming Yang, Jaime Carbonell, Ruslan Salakhutdinov, and Quoc V. Le. XLNet: Generalized Autoregressive Pretraining for Language Understanding. arXiv:1906.08237 [cs], January 2020. arXiv: 1906.08237.

29. Baris E. Suzek, Yuqi Wang, Hongzhan Huang, Peter B. McGarvey, Cathy H. Wu, and UniProt Consortium. UniRef clusters: a comprehensive and scalable alternative for improving sequence similarity searches. Bioinformatics (Oxford, England), 31(6):926-932, March 2015. ISSN 1367-4811. doi: 10.1093/bioinformatics/btu739.

30. Ying Huang, Beifang Niu, Ying Gao, Limin Fu, and Weizhong Li. CD-HIT Suite: a web server for clustering and comparing biological sequences. Bioinformatics, 26(5):680-682, March 2010. ISSN 1367-4803. doi: 10.1093/bioinformatics/btq003.

31. Martin Steinegger and Johannes Söding. Clustering huge protein sequence sets in linear time | Nature Communications, March 2018.

32. Zihang Dai, Zhilin Yang, Yiming Yang, Jaime Carbonell, Quoc V. Le, and Ruslan Salakhutdinov. Transformer-XL: Attentive Language Models Beyond a Fixed-Length Context. arXiv:1901.02860 [cs, stat], June 2019. arXiv: 1901.02860.

33. Ahmed Elnaggar, Michael Heinzinger, Christian Dallago, Ghalia Rehawi, Yu Wang, Llion Jones, Tom Gibbs, Tamas Feher, Christoph Angerer, Martin Steinegger, Debsindhu Bhowmik, and Burkhard Rost. ProtTrans: Towards Cracking the Language of Life's Code Through Self-Supervised Learning. Technical report, May 2021. Company: Cold Spring Harbor Laboratory Distributor: Cold Spring Harbor Laboratory Label: Cold Spring Harbor Laboratory Section: New Results Type: article.

34. Jie Hou, Badri Adhikari, and Jianlin Cheng. DeepSF: deep convolutional neural network for mapping protein sequences to folds. Bioinformatics, 34(8):1295-1303, April 2018. ISSN 1367-4803. doi: 10.1093/bioinformatics/btx780.

35. Hao Zhang, Chunhe Zhang, Zhi Li, Cong Li, Xu Wei, Borui Zhang, and Yuanning Liu. A New Method of RNA Secondary Structure Prediction Based on Convolutional Neural Network and Dynamic Programming. Frontiers in Genetics, 10:467, 2019. ISSN 1664-8021. doi: 10.3389/fgene.2019.00467.

36. Xinyue Kang, Fanyi Dong, Cheng Shi, Shicai Liu, Jian Sun, Jiaxin Chen, Haiqi Li, Hanmei Xu, Xingzhen Lao, and Heng Zheng. DRAMP 2.0, an updated data repository of antimicrobial peptides. Scientific Data, 6(1):148, August 2019. ISSN 2052-4463. doi: 10.1038/s41597-019-0154-y. Number: 1 Publisher: Nature Publishing Group.

37. Martın Abadi, Paul Barham, Jianmin Chen, Zhifeng Chen, Andy Davis, Jeffrey Dean, Matthieu Devin, Sanjay Ghemawat, Geoffrey Irving, Michael Isard, Manjunath Kudlur, Josh Levenberg, Rajat Monga, Sherry Moore, Derek G Murray, Benoit Steiner, Paul Tucker, Vijay Vasudevan, Pete Warden, Martin Wicke, Yuan Yu, and Xiaogiang Zheng. TensorFlow: A system for large-scale machine learning. page 21, 2016

38. Lisha Li, Kevin Jamieson, Giulia DeSalvo, Afshin Rostamizadeh, and Ameet Talwalkar. Hyperband: A Novel Bandit-Based Approach to Hyperparameter Optimization. March 2016.

39. Jasper Snoek, Hugo Larochelle, and Ryan P Adams. Practical Bayesian Optimization of Machine Learning Algorithms. In Advances in Neural Information Processing Systems, volume 25. Curran Associates, Inc., 2012.

40. Stefan Falkner, Aaron Klein, and Frank Hutter. BOHB: Robust and Efficient Hyperparameter Optimization at Scale. arXiv:1807.01774 [CS, stat], July 2018. arXiv: 1807.01774. 


\section{Supplementary Information}

\section{Sequence Length Distributions - LMPred Dataset:}

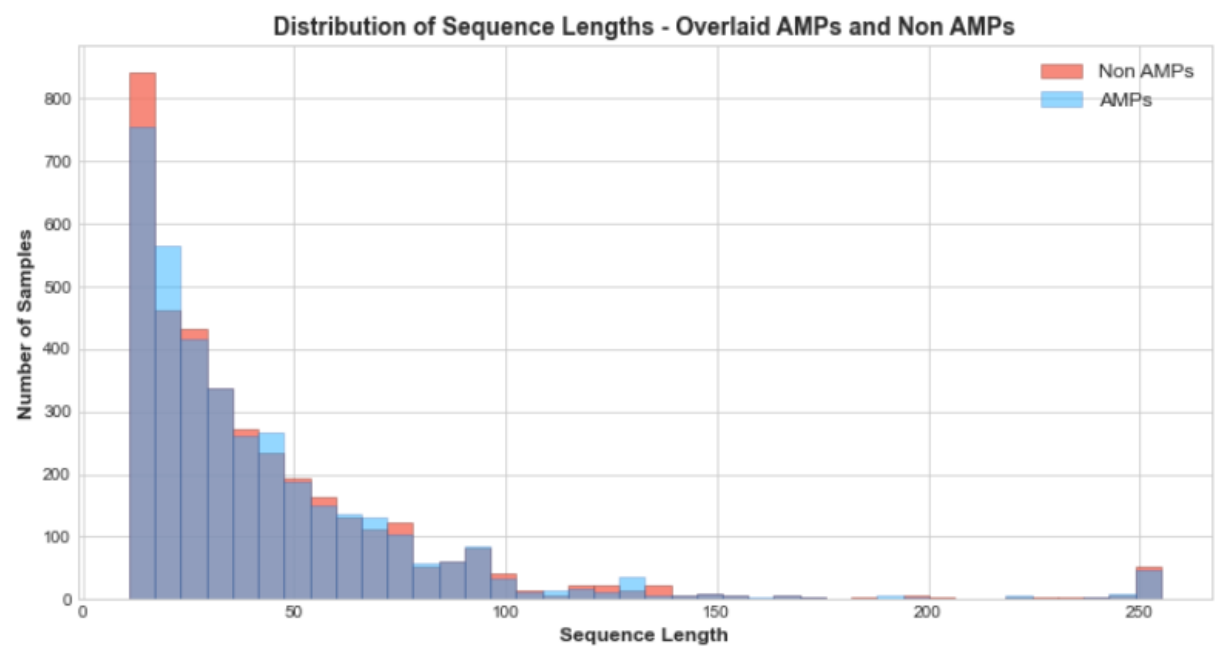

Figure S1: Sequence length distributions of the 3,758 AMP (blue) and 3,758 non-AMP (red) samples in the full LMPred dataset.
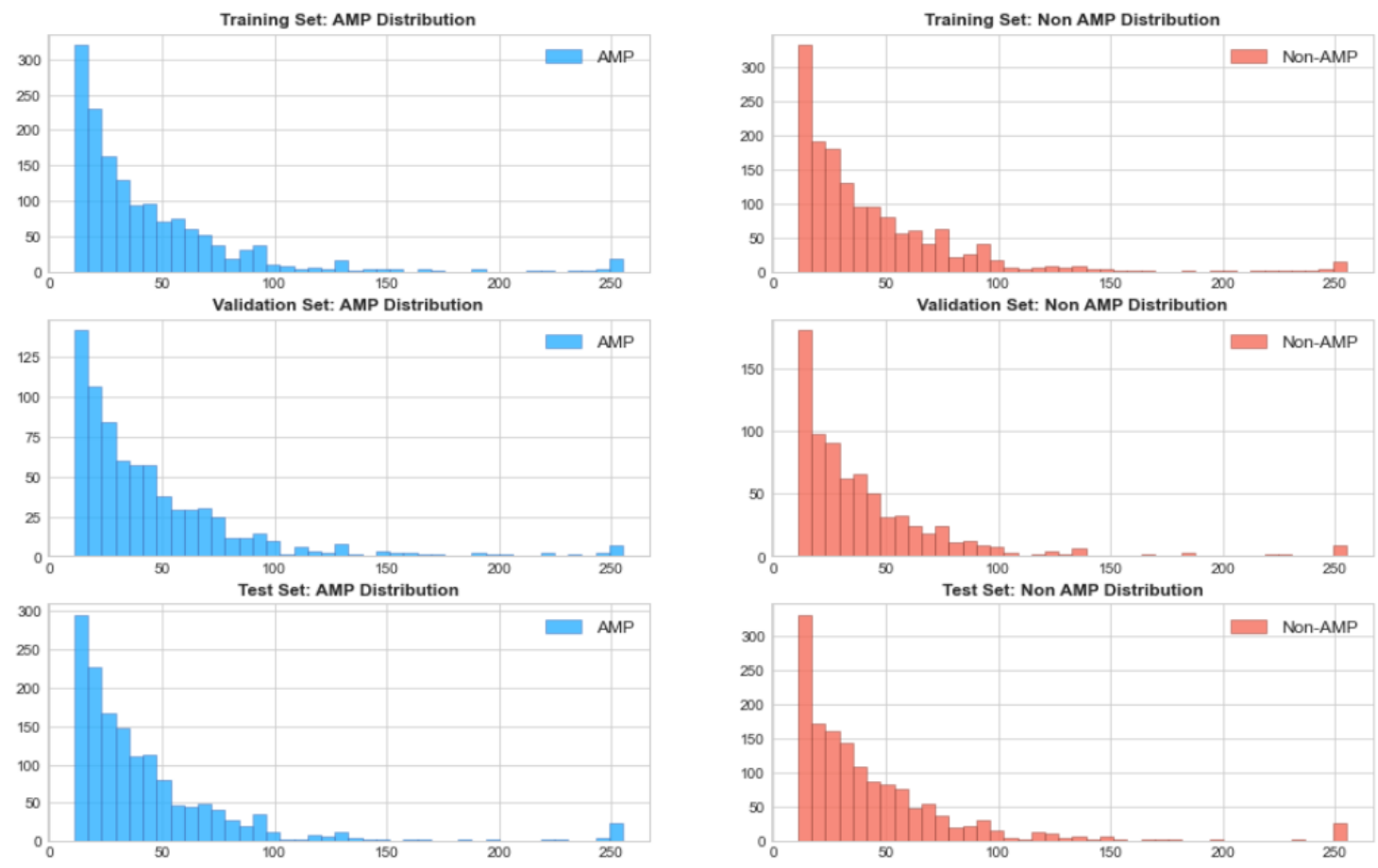

Figure S2: Sequence length distributions of the AMPs and non-AMPs across the training $(40 \%)$, validation (40\%) and test (20\%) datasets.

\section{Mean Embedding Values}

Below shows some evidence of the different information conveyed by the embeddings produced by the different pre-trained language models. This is most obvious when looking at 
the embedding of " $L$ " in position 11 of the non-AMP sample, compared to it's embedding in position 10 of the AMP sample. This difference is most stark for the T5 models where " $L$ " is given the average value of -0.002 in the non-AMP and -0.010 in the AMP position. Overall, the T5 models appear to display the most absolute variance between embeddings.

\section{Non-AMP Sequence}

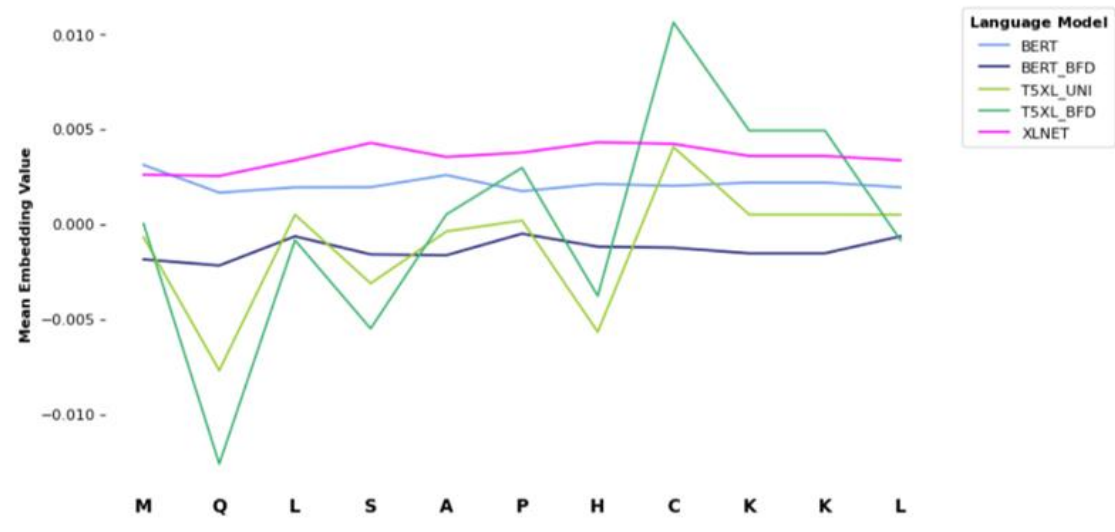

$\underline{\text { AMP Sequence }}$

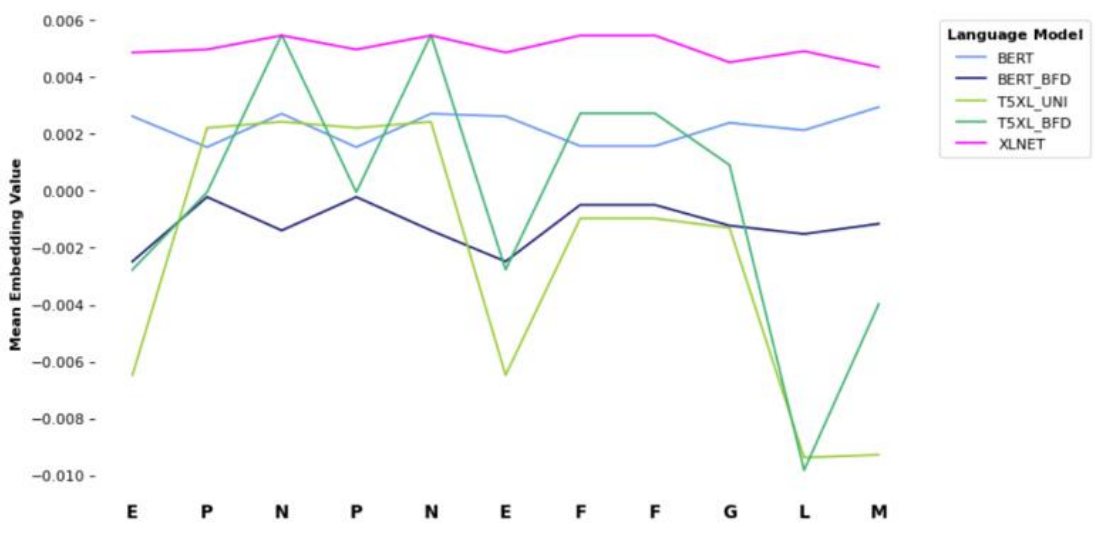

Figure S3: Line plots showing the comparative average value per amino acid assigned by the different pre-trained language models to an AMP and non-AMP 11 amino acids in length.

\section{Data Availability}

The LMPred dataset, along with notebooks detailing how the research can be replicated, can be found at the following Github page:

https://github.com/williamdee1/LMPred AMP Dataset 